\title{
3D content creation exploiting 2D character animation
}

\author{
Simone Barbieri \\ Centre for Digital Entertainment \\ Bournemouth University \\ Thud Media \\ sbarbieri@bournemouth.ac.uk
}

\author{
Tao Jiang \\ Bournemouth University \\ tjiang@bournemouth.ac.uk
}

\author{
Ben Cawthorne \\ Thud Media \\ ben.cawthorne@thudmedia.com
}

\author{
Zhidong Xiao \\ Bournemouth University \\ zxiao@bournemouth.ac.uk
}

\author{
Xiaosong Yang \\ Bournemouth University \\ xyang@bournemouth.ac.uk
}

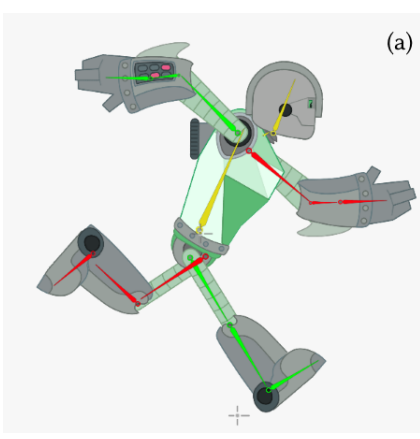

(a)

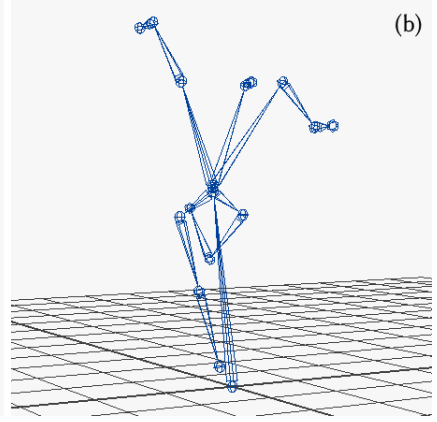

(b)

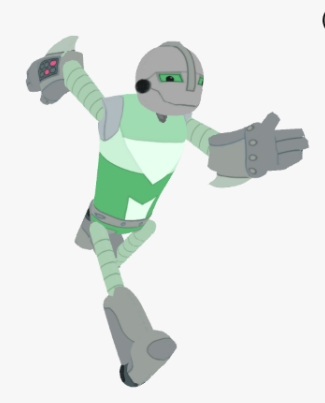

(c)

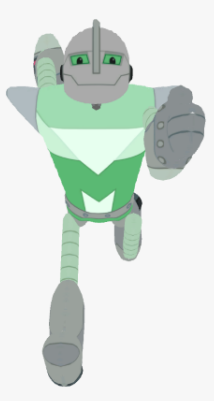

(d)

Figure 1: Stages of the generation of a $3 D$ character animation from a frame of the input $2 \mathrm{D}$ animation (a). (b) shows the computed 3D skeleton. (c) and (d) show a frame of the converted 3D animation from the side and from the front. $\odot$ Thud Media

\section{CCS CONCEPTS}

- Computing methodologies $\rightarrow$ Animation; Shape modeling;

\section{KEYWORDS}

animation, modeling, surface registration, 3D representation

\section{ACM Reference format:}

Simone Barbieri, Tao Jiang, Ben Cawthorne, Zhidong Xiao, and Xiaosong Yang. 2017. 3D content creation exploiting 2D character animation. In Proceedings of Conference Name, Conference Location, Conference Date and Year, 2 pages.

https://doi.org/10.1145/8888888.7777777

\section{INTRODUCTION}

While 3D animation is constantly increasing its popularity, 2D is still largely in use in animation production. In fact, 2D has two main advantages. The first one is economic, as it is more rapid to produce, having a dimension less to consider. The second one is important for the artists, as 2D characters usually have highly distinctive traits, which are lost in a 3D transposition. An iconic

Permission to make digital or hard copies of part or all of this work for personal or classroom use is granted without fee provided that copies are not made or distributed for profit or commercial advantage and that copies bear this notice and the full citation on the first page. Copyrights for third-party components of this work must be honored.

For all other uses, contact the owner/author(s).

Conference Name, Conference Date and Year, Conference Location

(C) 2017 Copyright held by the owner/author(s).

ACM ISBN 978-1-4503-1234-5/17/07 ..\$15.00

https://doi.org/10.1145/8888888.7777777 example is Mickey Mouse, whom ears appear circular no matter which way he is facing.

While other systems [Kitamura et al. 2015; Rivers et al. 2010] use $2.5 \mathrm{D}$ models, which move $2 \mathrm{D}$ elements in $3 \mathrm{D}$ to simulate the three-dimensionality of the characters, in this paper a new system is proposed for the generation of 3D content by using existing 2D character animations, and therefore could be easily integrated into the current production pipeline. In fact, the system is fully automatic and all the assets required to work usually are directly available from the $2 \mathrm{D}$ animation production pipeline. Minimal intervention is required only if the body parts (figure 2), are not provided from multiple perspectives. The aim of the system is to maintain the characteristics of the $2 \mathrm{D}$ character in the $3 \mathrm{D}$ environment.

\section{METHODOLOGY}

\subsection{Generation of the 3D model}

The first stage of our system is the modelling of the 3D character. To maintain it as similar as possible to its $2 \mathrm{D}$ version, an approximated $3 \mathrm{D}$ model is generated for each provided 2D image of the character's turnaround.

First, a 2D mesh is generated for each body part of the character. Then, according to [Olsen et al. 2011], the flat mesh is inflated by using the distance of each point from the contour. The inflated mesh is then mirrored to obtain a closed 3D mesh. Figure 3 shows this process. The generated $3 \mathrm{D}$ components are then combined to form the complete $3 \mathrm{D}$ character. If the character has been provided drawn from multiple perspectives, along with the 2D skeleton for each point of view, the system places each body part in 3D. If only 


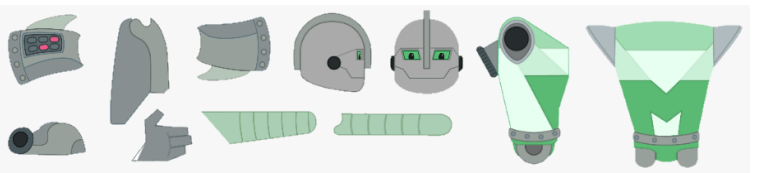

Figure 2: The character is provided split into several body parts from the existing pipeline. Otherwise, a minimal manual effort is required. @Thud Media

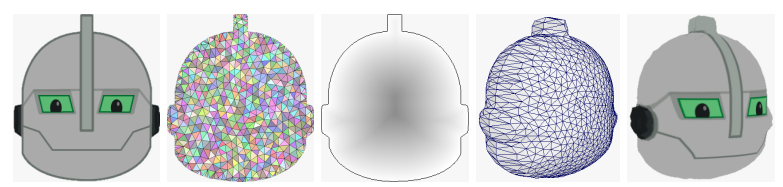

Figure 3: The modelling process of the system. @Thud Media

one image and one 2D skeleton have been provided, then user input is required.

\subsection{Surface registration}

For the body parts for which the user has provided multiple perspectives, to maintain all the character's features visible from different perspectives, a novel non-isometric surface registration method is proposed, inspired by the work of [Jiang et al. 2017].

Given a template mesh $\mathcal{S}$ and a target model $\mathcal{T}$, the goal is to deform the template $\mathcal{S}$ gradually into $\mathcal{S}^{\prime}$ so that the deformed mesh $\mathcal{S}^{\prime}$ is sufficiently close to the target $\mathcal{T}$ with structure preserved and feature points matched. Let $p, p^{\prime}, q$ denote the vertex positions on $\mathcal{S}, \mathcal{S}^{\prime}, \mathcal{T}$ respectively, the registration energy is defined as:

$$
E\left(p^{\prime}\right)=w_{l} E_{l}\left(p^{\prime}\right)+w_{d} E_{d}\left(p^{\prime}\right)+w_{c} E_{c}\left(p^{\prime}\right)+w_{f} E_{f}\left(p^{\prime}\right),
$$

where $E_{l}$ is the bi-Laplacian energy, $E_{d}$ is the consistent as-similaras-possible (CASAP) energy, $E_{c}$ is the correspondence constraint energy, and $E_{f}$ is the feature point constraint energy. The weights before each energy term adjust the influence they account for in the total energy.

The matching points between the two adjacent perspectives are computed before converting the images into 2D meshes. As in the current $2 \mathrm{D}$ animation pipeline the body parts are separate, feature points as the eyes or the mouth can be easily detected.

The registration is computed between all the couple of models generated from adjacent perspectives in both ways: using one as the template and the other as the target, and vice versa. In the system, the model of the closest perspective is shown. While the camera rotates around the character, the position of the vertices is interpolated between their position in the source model and in the registration. The registration result can be seen from the supplemental video.

\subsection{Generation of the 3D animation}

The system computes automatically a 3D skeleton only for the first frame of the animation, using the provided 2D skeletons. If only one side is provided, then the joints must be placed manually on the $z$ axis. To bind together the skeleton and the model, the system applies a rigid skinning.
Once obtained the 3D skeleton for the initial binding pose, the system computes the 3D animation by reconstructing the 3D poses for each keyframe of the 2D animation. Inspired by the works of [Barbieri et al. 2016], a new optimization method is introduced to convert the $2 \mathrm{D}$ animation. The $2 \mathrm{D}$ skeleton is sampled to obtain the set of $2 \mathrm{D}$ points $Y=\left(y_{1}, y_{2}, \ldots, y_{M}\right)$. The same sampling is applied to the 3D skeleton, to obtain the same number of points $V=$ $\left(v_{1}, v_{2}, \ldots, v_{M}\right)$ and thus a one-to-one correspondence between the points in the two sets.

The objective is to find the rigging parameters $p$ which deform the points in $V$ as closely as possible to those in $Y$. The optimization problem is defined as:

$$
\underset{p}{\arg \min } \sum_{i=1}^{M}\left\|y_{i}-\mathbf{C} \cdot v_{i}(p)\right\|_{2}^{2}+\Phi(p)
$$

where $\mathbf{C} \in \mathbb{R}^{2 \times 3}$ is the camera projection matrix, which is used to compare the points in $Y$ with the projection of the points in $V$. $\Phi(p)$ is a regularization term which have two purposes: to penalise solutions without the least amount of change compared with the initial pose and to promote solutions which keep the deformed point in $V$ in the same viewing plane.

The optimization problem is solved for each keyframe, while the other frames are interpolated.

\section{CONCLUSIONS}

In the paper, a new system to generate 3D content by exploiting $2 \mathrm{D}$ character animations has been presented. The system is able to generate a $3 \mathrm{D}$ character from $2 \mathrm{D}$ images, with a minimum intervention of the drawer in the input drawings. Moreover, it is able to convert 2D animations into 3D automatically, allowing the users to repurpose old animations or to keep working with 2D. Figure 1 shows a frame of a running animation of a $2 \mathrm{D}$ character and the generated 3D version from two different points of view. Additionally, the supplemental video shows an overview of the entire process.

Our system not only has the advantage to keep the converted $2 \mathrm{D}$ characters highly recognizable even in 3D, due to the applied registration method, but also has the economic advantage to reuse drawing and animations from their $2 \mathrm{D}$ versions. There are several application for our system. First, it could be used to repurpose 2D character animations from animated film or shows to make video games or other kind of 3D applications. Secondly, it could be used to bring 2D characters into Virtual or Augmented Reality, which are increasingly popular.

\section{REFERENCES}

Simone Barbieri, Nicola Garau, Wenyu Hu, Zhidong Xiao, and Xiaosong Yang. 2016. Enhancing character posing by a sketch-based interaction. In ACM SIGGRAPH 2016 Posters. ACM, 56. https://doi.org/10.1145/2945078.2945134

Tao Jiang, Kun Qian, Shuang Liu, Jing Wang, Xiaosong Yang, and Jianjun Zhang. 2017. Consistent as-similar-as-possible non-isometric surface registration. The Visual Computer (2017), 1-11. https://doi.org/10.1007/s00371-017-1390-9

Maki Kitamura, Yoshihiro Kanamori, and Reiji Tsuruno. 2015. 2.5 D Modeling from Illustrations of Different Views. 8, 4 (2015), 74-79.

Luke Olsen, Faramarz Samavati, and Joaquim Jorge. 2011. Naturasketch: Modeling from images and natural sketches. IEEE Computer Graphics and Applications 31, 6 (2011), 24-34. https://doi.org/10.1109/mcg.2011.84

Alec Rivers, Takeo Igarashi, and FrÃldo Durand. 2010. 2.5 D cartoon models. In ACM Transactions on Graphics (TOG), Vol. 29. ACM, 59. https://doi.org/10.1145/ 1833351.1778796 